\title{
Mitteilungen der Sektion Internationale Politik
}

\author{
1. Ergebnisse der Mitgliederversammlung im Rahmen des \\ DVPW-Kongresses 2003
}

Im Mittelpunkt der Mitgliederversammlung stand die Diskussion der über die Mailing-Liste verschickten Tätigkeitsberichte des Vorstands sowie Neuwahlen. Zu den wichtigsten Ergebnissen der Arbeit der Sektion in den vergangenen drei Jahren gehören zwei Sammelbände über »Die neuen Internationalen Beziehungen« (hrsg. von Gunther Hellmann, Klaus Dieter Wolf und Michael Zürn) sowie »Global Economic Governance« (hrsg. von Stefan Schirm). Beide Bände resultierten aus thematisch fokussierten Sektionstagungen. In der Diskussion wurde kritisch angemerkt, dass die Produktion kohärenter Sammelbände zwar helfe, die Binnenkommunikation unter denjenigen zu fördern, die in demselben Feld arbeiten. Die ausschließliche Konzentration der Sektionsarbeit auf thematisch eng definierte Sektionstagungen drohe allerdings, wichtige Segmente der Sektion zu vernachlässigen, die bei thematisch offenen Tagungen eher zum Zuge kommen könnten. Zudem würde dadurch eine wichtige potenzielle Quelle für Manuskripte ausgetrocknet, die bei der ZIB eingereicht werden könnten. Der neue Sektionsvorstand wird diese Anregungen aufgreifen und in den nächsten drei Jahren eine Tagung organisieren, die für unterschiedliche Felder offen ist.

Bei den Neuwahlen des Sektionsvorstandes wurden Gunther Hellmann, Peter Rudolf und Frank Schimmelfennig gewählt. Die Geschäftsführung 2003/2004 wird in Frankfurt angesiedelt sein. Neben der bereits erwähnten Tagung mit offener Aus-

schreibung plant der neue Vorstand eine Tagung, die sich u. a. aus der Perspektive der neueren Wissenschaftsforschung mit dem Verhältnis von Wissenschaft und Praxis bzw. Wissenschaft und Öffentlichkeit beschäftigen wird. Auf der Homepage der Sektion werden sich hierzu Anfang 2004 weitere Details finden. Der Vorstand ist offen für Anregungen zur Ausgestaltung beider Tagungen und bittet, entsprechende Rückmeldungen bzw. Vorschläge direkt an die Geschäftsführung in Frankfurt zu richten.

\section{Adressen- und Mailing-Liste/Aktualisierung}

Mitglieder der DVPW, die sich in ihren Arbeits- bzw. Interessenschwerpunkten der Sektion »Internationale Politik« zurechnen, sollten dies dem Sektionsvorstand mitteilen. Sie werden dann in die Adressen- und Mailing-Liste der Sektion aufgenommen. Die aktuelle Liste der Sektionsmitglieder ist über die Homepage der Sektion unter http://www.soz.uni-frankfurt.de/hellmann/sektion/ zugänglich. Wir möchten die Mitglieder der Sektion bitten, ihre Adressdaten, insbesondere aber die in der 
Adressenliste aufgeführte E-Mail-Adresse zu überprüfen, da die Kommunikation zwischen Vorstand und Sektion zunehmend über die Mailing-Liste erfolgt. Adressergänzungen bzw. -korrekturen sollten dem jeweils geschäftsführenden Vorstandsmitglied mitgeteilt werden.

\section{Tagung der Nachwuchsgruppe der Sektion Internationale Politik}

Die Nachwuchsgruppe der Sektion Internationale Politik (IP) der Deutschen Vereinigung für Politische Wissenschaft (DVPW) veranstaltet vom 30. Januar bis 1. Februar 2004 im Studienhaus Wiesneck in Buchenbach bei Freiburg i. Brsg. zum Thema »Sicherheit, Wirtschaft, Gesellschaft: Theorien und Problemfelder internationaler Beziehungen« die sechste Tagung für den wissenschaftlichen Nachwuchs. Die Tagung gibt Nachwuchswissenschaftlerinnen und -wissenschaftlern die Gelegenheit, ihre abgeschlossenen Magisterarbeiten oder in Bearbeitung befindlichen Dissertationsprojekte vorzustellen und mit einem interessierten Fachpublikum zu diskutieren. Wir möchten nochmals darauf hinweisen, dass die Tagung auch Studierenden offen steht, die selbst nicht vorstellen werden, aber Interesse am wissenschaftlichen Austausch haben.

Für aktuelle Informationen zur Nachwuchstagung besuchen Sie bitte unsere neue Homepage, die unter der URL: http://www.uni-frankfurt.de/fb03/ib-nachwuchsgruppe/ ab Januar 2004 zur Verfügung steht.

Rainer Baumann

Johann Wolfgang Goethe-Universität Institut für Vergleichende Politikwissenschaft und Internationale Beziehungen

Robert-Mayer-Straße 5, Fach 102

D-60054 Frankfurt am Main

Tel. 069/798 28488

Fax 069/79828460

r.baumann@soz.uni-frankfurt.de
Dr. Anja Jetschke

Seminar für Wissenschaftliche Politik Albert-Ludwigs-Universität Freiburg

Rempartstraße 15

79085 Freiburg

Tel. 0761/203 3470

Fax: 0761/203 9185

anja.jetschke@politik.uni-freiburg.de

Für Rückfragen stehen die Sprecher der Sektion unter folgenden Adressen zur Verfügung:

Prof. Dr. Gunther Hellmann

(Geschäftsführung vom 1.10.2003 bis 30.9.2004)

Johann-Wolfgang-Goethe-Universität Frankfurt am Main

Institut für Vergleichende Politikwissenschaft und Internationale Beziehungen

Robert-Mayer-Straße 5, Fach 102

60054 Frankfurt a. M.

Tel.: 069/798-25191 oder -22667

g.hellmann@soz.uni-frankfurt.de

http://www.soz.uni-frankfurt.de/hellmann/start.htm 
PD Dr. Peter Rudolf

Stiftung Wissenschaft und Politik

Ludwigkirchplatz 3-4

10719 Berlin

Tel.: 030/88007-242

peter.rudolf@swp-berlin.org

http://www.swp-berlin.org/mitarb/rdf.html

PD Dr. Frank Schimmelfennig

Universität Mannheim

Mannheimer Zentrum für Europäische Sozialforschung

68131 Mannheim

Tel.: 0621/181-2813

Fax: 0621/181-2845

frank.schimmelfennig@mzes.uni.mannheim.de

http://www.mzes.uni-mannheim.de/users/schimmelfennig/Homepage.html 